\title{
Giant pseudomeningocele causing urinary obstruction in a patient with Marfan syndrome
}

\author{
Jeremy G. Stone, MD, ' Liisa L. Bergmann, MD, ${ }^{1}$ Ryan Takamori, MD, ${ }^{2}$ and Daniel J. Donovan, MD¹ \\ 'Department of Surgery, University of Hawaii, John A. Burns School of Medicine; and 'Department of Surgery, Kaiser \\ Permanente Moanalua Medical Center, Honolulu, Hawaii
}

Defective collagen biosynthesis in Marfan syndrome predisposes to dural defects such as dural ectasia, meningocele, and pseudomeningocele; thus, an increased index of suspicion for these conditions should be present in the clinical setting of Marfan syndrome. The authors describe a young woman with Marfan syndrome who was being treated with anticoagulants for a prosthetic heart valve and who presented with a spontaneous retroperitoneal hemorrhage requiring surgical evacuation. No CSF leak was encountered at surgery, but she developed progressively more severe positional headaches over the following year. She then experienced the sudden onset of acute urinary obstruction, at which time CT revealed a $17 \times 15 \times 13-\mathrm{cm}$ presacral pseudomeningocele communicating with the thecal sac through a sacral bone defect. An anterior surgical approach was used for drainage of the pseudomeningocele as well as for primary closure of the dural defect with a bovine pericardial patch and autologous subcutaneous fat graft. After a short period of lumbar subarachnoid drainage of the CSF, the patient was able to resume normal activity without recurrent symptoms. To the authors' knowledge, such a pseudomeningocele in a patient with Marfan syndrome has been reported only twice, and this case features the largest pseudomeningocele to date. They also review the pertinent literature regarding presentation, diagnosis, and management of these lesions.

http://thejns.org/doi/abs/10.3171/2014.11.SPINE131086

KEY WORDS pseudomeningocele; Marfan syndrome; anterior; sacral; surgery

$\mathrm{P}$ SEUDOMENINGOCELES are extradural collections of CSF resulting from an abnormal opening in the dura and arachnoid layers, and spontaneous ones are rarely encountered. They are most commonly iatrogenic, occurring after incidental durotomy during posterior decompression surgery and complicating up to $2 \%$ of spinal procedures. ${ }^{8,16}$ Pseudomeningoceles can also arise in the setting of blunt or penetrating spinal trauma ${ }^{6}$ Congenital pseudomeningoceles can be either posterior or anterior, but most anterior pseudomeningoceles are thought to be congenital. These lesions often occur in the setting of connective tissue diseases, such as Marfan syndrome, and are also associated with neurofibromatosis. ${ }^{3}$ We report the unusual case of a woman with Marfan syndrome and a giant anterior sacral pseudomeningocele that caused urinary obstruction. This case is only the third reported instance of a pseudomeningocele in a patient with Marfan syndrome, and the lesion is the largest described to date. ${ }^{7,14}$

\section{Case Report}

History and Examination

A 21-year-old woman with Marfan syndrome was on chronic anticoagulants for aortic and mitral valve replacement and had undergone spinal T1-L4 segment fusion for scoliosis 5 years earlier. She presented with the acute onset of atraumatic abdominal pain and was found to have a large retroperitoneal hematoma on CT. Further review showed an excellent position of all her spinal hardware, without penetration of the thecal sac or anteriorly through the bone. She underwent exploratory laparotomy and evacuation of the hematoma, without findings of malignancy or any other obvious source of bleeding. No CSF leak was encountered, and no bone was removed from the sacrum or other portions of the spine. She then experienced the gradual onset of positional headaches, low-back pain, nausea, and emesis over the course of the following

SUBMITTED December 5, 2013. ACCEPTED November 25, 2014

INCLUDE WHEN CITING Published online April 24, 2015; DOI: 10.3171/2014.11.SPINE131086.

DISCLOSURE The authors report no conflict of interest concerning the materials or methods used in this study or the findings specified in this paper. 
year, and her condition was refractory to medications and without a definitive diagnosis. Fourteen months after her initial surgery, she experienced acute urinary retention, and CT studies of the abdomen and pelvis revealed a large pelvic fluid collection obstructing the ureters bilaterally and measuring $17 \times 15 \times 13 \mathrm{~cm}$ (Fig. 1). The fluid-filled mass was contiguous with the left S-1 nerve root sleeve and extended via the neural foramen to communicate with the thecal sac. The uterus and urinary bladder were compressed anteriorly, and the patient had severe hydroureteronephrosis, but bilateral ureteral stent placement immediately resolved the urinary retention. Magnetic resonance imaging studies of the lumbosacral spine were obtained, but because there was a severe artifact due to the stainless steel instrumentation placed by another surgeon for her previous scoliosis repair, the studies were not helpful in delineating the anatomy. Follow-up myelography and subsequent CT confirmed communication between the fluid collection and lumbosacral spinal canal, confirming a pseudomeningocele and prompting surgical repair.

She was a thin woman with Marfanoid body habitus, appeared uncomfortable whenever sitting upright, and had to lie down frequently because of headache. Her cranial nerves were intact. Lower extremity sensation was intact, but there was trace weakness in the plantar flexors of her left ankle and a decreased ankle jerk on the left compared with the right. Her abdomen was mildly distended, without tenderness.

\section{Operation}

The patient was admitted for 3 days of anticoagulation bridge on a heparin drip, which was stopped the morning of surgery. A lumbar subarachnoid drain was placed at the L4-5 interspace, and an anterior approach was used, with her abdomen opened via the existing incisional scar. A robust membrane was freed from adherent bowel, and $1.5 \mathrm{~L}$ of spinal fluid was removed from the mass immediately. A clear defect leaking spinal fluid was directly visualized on the left side of the superior aspect of the sacrum, with several openings in the dura that together measured $1.3 \times 2.0$ $\mathrm{cm}$ (Fig. 2). These dural septations were divided so that one large opening was created. The opening was closed with autologous fat and a bovine pericardial patch, which were sutured in place, and then covered with Surgicel, Gelfoam, and tissue sealant.

\section{Postoperative Course}

Her postoperative course was uneventful, with continued lumbar subarachnoid drainage for a total of 5 days and hospital discharge on postoperative Day 12 after a heparin to warfarin bridge was completed. Her headaches resolved completely and she resumed normal activities without symptoms. Postoperative CT a month later showed a collapsed pseudomeningocele and bilateral ureteral stents with resolution of the bilateral hydronephrosis and hydroureter (Fig. 3). She had had no recurrence of her headaches or other symptoms at the 30-month clinical follow-up, but she has declined to undergo repeat imaging despite several requests.
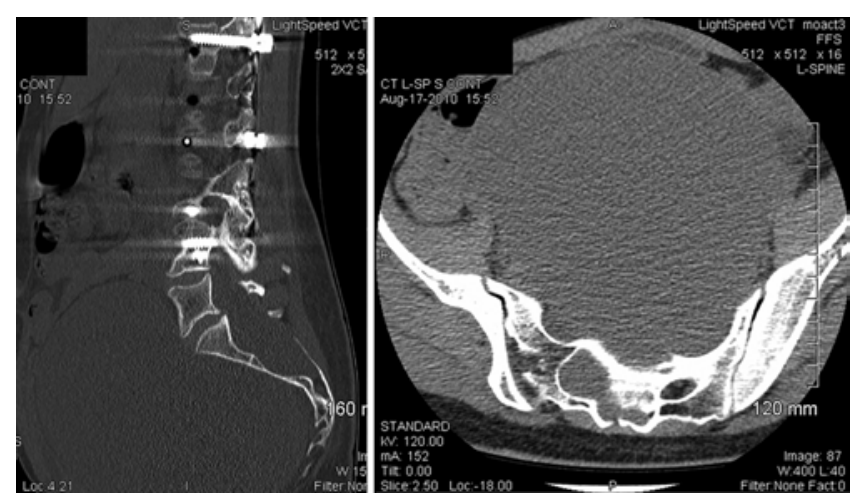

FIG. 1. Preoperative sagittal reconstruction (left) and axial (right) CT scans obtained postmyelography, demonstrating a large sacral pseudomeningocele with compression of the pelvic organs.

\section{Discussion}

Although spontaneous pseudomeningocele is a rare condition, a higher index of clinical suspicion is needed in patients with Marfan syndrome. In this syndrome, a fibrillin-1 gene mutation inherited in an autosomal dominant fashion results in defective fibrillin-1 secretion and causes subsequent weakening of the microfilaments in elastic fibers. ${ }^{4}$ In the setting of a more elastic dura (dural ectasia), meningoceles arise over time because of normal CSF pulsations. In some cases, rupture through the dura creates a pseudomeningocele, which can fill the potential adjacent spaces, including large ones such as the pelvis, as seen in the featured case.

Pseudomeningocele can present with diverse clinical symptoms and findings depending on its location and size. Most often, localized back pain and postural headaches are presenting symptoms. In the case of large pseudomeningoceles, postural headache can result from shifting CSF volumes and intracranial hypotension in the upright position, which cause traction on the meninges and blood vessels. Rarely, nerve root entrapment in the dural opening can produce radicular symptoms; and in very rare and extreme cases, spinal cord herniation can result in progressive myelopathy. ${ }^{1,5}$ As in the featured case, sacral pseudomeningocele can compress pelvic or intraabdominal organs such as the ureters, bladder, rectum, or uterus. When pseudomeningocele is suspected, MRI is the imaging test of choice given its optimal soft tissue visualization and ability to identify the tract of communication with the
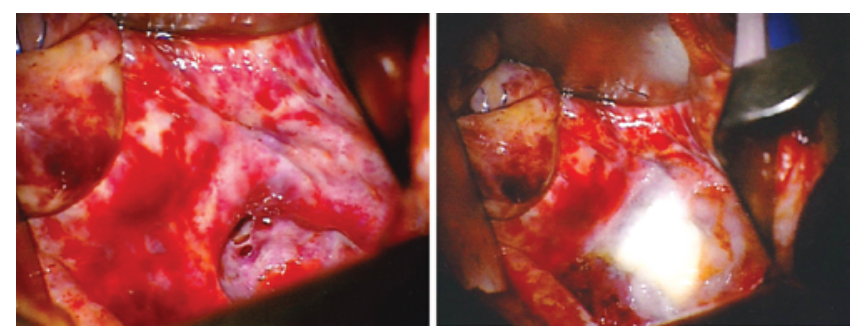

FIG. 2. Intraoperative images. A dural defect located at the left S-1 neural foramen with multiple septations is evident (left), and primary closure of the dural defect was achieved with an autologous subcutaneous fat graft, a bovine pericardial patch, Surgicel, Gelfoam, and tissue sealant (right). 


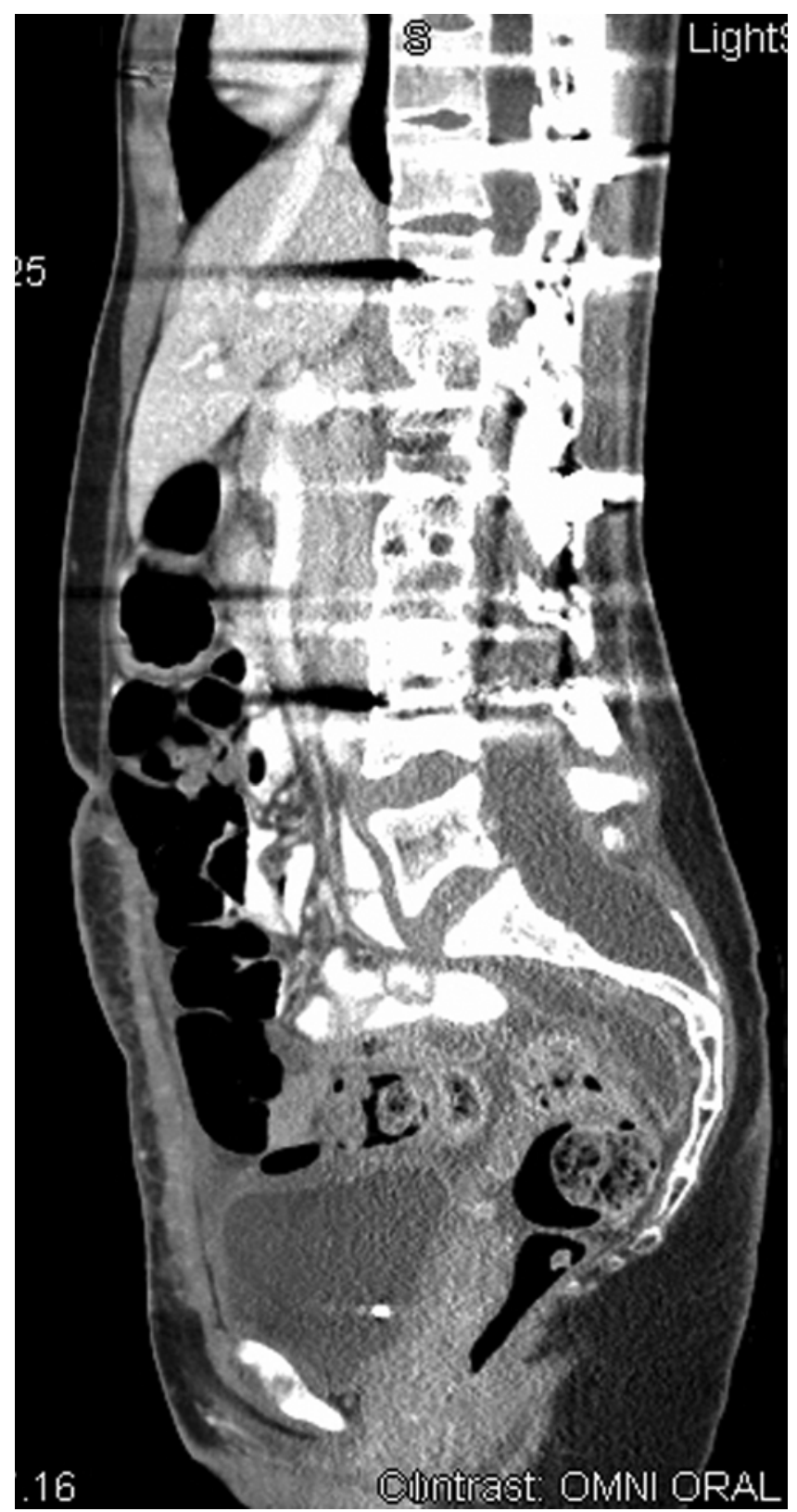

FIG. 3. Postoperative imaging. Sagittal reconstruction CT confirmed resolution of the pseudomeningocele and return of the pelvic contents to their normal anatomical location.

thecal sac. However, CT myelography, myelography, and retrograde radionucleotide myelography can be useful in individual cases to confirm the diagnosis, especially with smaller CSF collections. ${ }^{2}$

Appropriate treatment of pseudomeningoceles requires an individual approach and most likely will involve some combination of surgical and nonsurgical options. Nonsurgical measures to consider include bed rest, focal compression, and abdominal binders., ${ }^{9,16}$ An epidural blood patch is a well-described option that has been effectively combined with fibrin glue to treat pseudomeningocele. ${ }^{10}$ Diversion of CSF by a closed subarachnoid drain several levels above a lumbar pseudomeningocele can also be a helpful adjuvant measure. ${ }^{13}$ However, in the setting of pseudomeningoceles that are symptomatic, large, and anterior or when conservative management fails, definitive surgical dural repair is indicated..$^{17}$

The principles of surgical repair include careful delineation of the pseudomeningocele capsule, entrance into and drainage of the CSF, reduction of any neural elements within the dura, and primary closure of the dural defect. A variety of agents have been described to aid in primary closure, including myofascial patch, autologous fat, cadaveric dura, pericardial patch, fibrin glue, and Gelfoam, ${ }^{11,17}$ and we used most of these in our repair. Some authors have described successful repair with a laparoscopic approach alone, but this has only been reported for a very small fistulous connection ${ }^{12}$ or for simple drainage without definitive repair, ${ }^{15}$ and even those who have used the laparoscopic technique have stated that it is contraindicated in the presence of a large anterior sac. ${ }^{15}$ Because of the pseudomeningocele's very large size and its association with pelvic and abdominal contents in our case, we selected an open anterior approach as the best exposure. Weng et al. described success in a series of postsurgical giant pseudomeningoceles (not involving Marfan syndrome) through the use of a combined treatment of open revision and extirpation, repair of dural tears, and implantation of a subarachnoid catheter for drainage. ${ }^{17}$ We used this combined approach with success in our patient and would recommend it to others for definitive repair and the removal of mass effect in the pelvis and/or abdomen.

\section{Acknowledgments}

We acknowledge the assistance of Ms. Karen Chang in the review of this manuscript and Lori Jennings, CHRC, Research Compliance Manager at Kaiser Moanalua Medical Center.

\section{References}

1. Cobb C III, Ehni G: Herniation of the spinal cord into an iatrogenic meningocele. Case report. J Neurosurg 39:533-536, 1973

2. Colletti PM, Siegel ME: Posttraumatic lumbar cerebrospinal fluid leak: detection by retrograde in-111-DTPA myeloscintography. Clin Nucl Med 6:403-404, 1981

3. Dolynchuk KN, Teskey J, West M: Intrathoracic meningocele associated with neurofibromatosis: case report. Neurosurgery 27:485-487, 1990

4. Fattori R, Nienaber CA, Descovich B, Ambrosetto P, Reggiani LB, Pepe G, et al: Importance of dural ectasia in phenotypic assessment of Marfan's syndrome. Lancet 354:910 913, 1999

5. Hadani M, Findler G, Knoler N, Tadmor R, Sahar A, Shacked I: Entrapped lumbar nerve root in pseudomeningocele after laminectomy: report of three cases. Neurosurgery 19:405-407, 1986

6. Hadley MN, Carter LP: Sacral fracture with pseudomeningocele and cerebrospinal fluid fistula: case report and review of the literature. Neurosurgery 16:843-846, 1985

7. Kohler E, Prentice D: Pseudomeningocele induced transient loss of consciousness in Marfan syndrome. Intern Med J 40:228-230, 2010

8. Lee KS, Hardy IM II: Postlaminectomy lumbar pseudomeningocele: report of four cases. Neurosurgery 30:111114, 1992

9. Leis AA, Leis JM, Leis JR: Pseudomeningoceles: a role for mechanical compression in the treatment of dural tears. Neurology 56:1116-1117, 2001 
10. McCormack BM, Taylor SL, Heath S, Scanlon J: Pseudomeningocele/CSF fistula in a patient with lumbar spinal implants treated with epidural blood patch and a brief course of closed subarachnoid drainage. A case report. Spine (Phila Pa 1976) 21:2273-2276, 1996

11. Pomeranz S, Constantini S, Umansky F: The use of fibrin sealant in cerebrospinal fluid leakage. Neurochirurgia (Stuttg) 34:166-169, 1991

12. Raftopoulos C, Pierard GE, Rétif C, Braude P, Brotchi J: Endoscopic cure of a giant sacral meningocele associated with Marfan's syndrome: case report. Neurosurgery 30:765-768, 1992

13. Shapiro SA, Scully T: Closed continuous drainage of cerebrospinal fluid via a lumbar subarachnoid catheter for treatment or prevention of cranial/spinal cerebrospinal fluid fistula. Neurosurgery 30:241-245, 1992

14. Sonier CB, Buhe T, Despins P, Delumeau J, de Kersaint-Gilly A: Sacral pseudomeningocele and Marfan's disease. One case. J Neuroradiol 20:292-296, 1993

15. Trapp C, Farage L, Clatterbuck RE, Romero FR, Rais-Bahrami S, Long DM, et al: Laparoscopic treatment of anterior sacral meningocele. Surg Neurol 68:443-448, 2007
16. Waisman M, Schweppe Y: Postoperative cerebrospinal fluid leakage after lumbar spine operations. Conservative treatment. Spine (Phila Pa 1976) 16:52-53, 1991

17. Weng YJ, Cheng CC, Li YY, Huang TJ, Hsu RW: Management of giant pseudomeningoceles after spinal surgery.

BMC Musculoskelet Disord 11:53, 2010

\section{Author Contributions}

Conception and design: all authors. Acquisition of data: all authors. Analysis and interpretation of data: all authors. Drafting the article: Donovan, Stone, Bergmann. Critically revising the article: Donovan, Stone, Bergmann. Reviewed submitted version of manuscript: all authors. Approved the final version of the manuscript on behalf of all authors: Donovan.

\section{Correspondence}

Daniel J. Donovan, Physicians Office Building I, 1380 Lusitana St., Ste. 1009, Honolulu, HI 96817. email: ddonovan-hng@ hawaii.rr.com. 